\title{
On Maximally Inflected Hyperbolic Curves
}

\author{
Aubin Arroyo • Erwan Brugallé • \\ Lucia López de Medrano
}

Received: 15 November 2013 / Revised: 13 May 2014 / Accepted: 19 May 2014 /

Published online: 2 July 2014

(C) Springer Science+Business Media New York 2014

\begin{abstract}
In this note we study the distribution of real inflection points among the ovals of a real non-singular hyperbolic curve of even degree. Using Hilbert's method we show that for any integers $d$ and $r$ such that $4 \leq r \leq 2 d^{2}-2 d$, there is a non-singular hyperbolic curve of degree $2 d$ in $\mathbb{R}^{2}$ with exactly $r$ line segments in the boundary of its convex hull. We also give a complete classification of possible distributions of inflection points among the ovals of a maximally inflected non-singular hyperbolic curve of degree 6.
\end{abstract}

Keywords Maximally inflected hyperbolic real curves and their convex hull . Patchworking of real algebraic curves · Tropical curves

\section{Introduction}

The fact that a non-singular real algebraic curve in $\mathbb{R P}^{2}$ of degree $\delta$ has at most $\delta(\delta-2)$ real inflection points was proved by Klein in 1876 , see [5]; see also [9,10] and [13]. A non-singular real plane algebraic curve is called maximally inflected if it has $\delta(\delta-2)$ distinct real inflection points. The existence of maximally inflected curves of any degree was also proved by Klein. Possible distributions of inflection points of a

\footnotetext{
A. Arroyo · L. L. de Medrano

Unidad Cuernavaca del Instituto de Matemáticas, Universidad Nacional Autonoma de México, Cuernavaca, Mexico

e-mail: lucia.1dm@im.unam.mx

A. Arroyo

e-mail: aubinarroyo@im.unam.mx

E. Brugallé

Centre Mathématiques Laurent Schwatrz, École Polytechnique, 91128 Palaiseau Cedex, France

e-mail: erwan.brugalle@math.cnrs.fr
} 
maximally inflected real algebraic curve in $\mathbb{R P}^{2}$ are subject to non-trivial obstructions that mainly remain mysterious (see for example [4] and [2]). In this note we focus on the case of maximally inflected hyperbolic curves of even degree.

Let $X=\mathbb{R}^{2}$ or $\mathbb{R P}^{2}$. A real algebraic curve $C$ in $X$ is said to be hyperbolic if there exists a point $p \in X \backslash C$ such that any real line through $p$ intersects $C$ only in real points. The topology of a non-singular hyperbolic curve is easy to describe. An embedded circle $O$ in $X$ is called an oval if its complement in $X$ has two connected components. In this case, one of them, called the interior of $O$ and denoted by $\operatorname{Int}(O)$, is homeomorphic to a disk, while the other is called the exterior of $O$ and denoted by $\operatorname{Ext}(O)$. An oval $O$ is said to be contained in another oval $\tilde{O}$ if $O \subset \operatorname{Int}(\tilde{O})$. A nonsingular hyperbolic curve $C$ of degree $2 d$ is a set of $d$ nested ovals, i.e. the inclusion relation among its ovals is a total ordering. The oval of $C$ containing all the others is called the outer oval of $C$. We define the interior and the exterior of $C$, denoted by Int $(C)$ and $\operatorname{Ext}(C)$, as the interior and exterior of its outer oval respectively.

\subsection{Line Segments in the Boundary of the Convex Hull of a Hyperbolic Curve in $\mathbb{R}^{2}$}

Denote by Hull ( $S$ ) the convex hull of a compact set $S \subset \mathbb{R}^{2}$. Of course, if $S$ is not convex then the boundary of Hull $(S)$ contain some line segments. Denote by $s(S)$ the number of line segments in $\partial \mathrm{Hull}(S)$.

In the case of a non-singular hyperbolic curve $C$ of degree $2 d$ in $\mathbb{R}^{2}$, the number $\mathrm{s}(C)$ is related to the number of inflection points contained in the outer oval $O$ of $C$. More precisely, for each line segment $l$ in $\partial \operatorname{Hull}(C)$, there are at least two inflection points of $C$ in the connected component of the closure $O \backslash \partial \mathrm{Hull}(C)$ with the same endpoints than $l$. Klein Inequality then implies that

$$
\mathrm{s}(C) \leq 2 d(d-1) \text {. }
$$

Next theorem shows, in particular, that this upper bound is sharp, answering to a question posed by De Loera et al. [3].

Theorem 1.1 Let $d$ and $r$ be two positive integers such that $4 \leq r \leq 2 d(d-1)$. Then there exists a non-singular maximally inflected hyperbolic curve $C$ of degree $2 d$ in $\mathbb{R}^{2}$ with $s(C)=r$.

The proof of Theorem 1.1 is given in Sect. 2, and is based on Hilbert's method of construction of real algebraic curves. See [14] for an exposition of this method in modern terms. For the reader's convenience, we break the proof of Theorem 1.1 into two parts: in Sect. 2.1 we explain how to adapt Hilbert's method to construct nonsingular maximally inflected hyperbolic curves of even degree with all real inflection points contained in the outer oval; in Sect. 2.2 we adapt this construction to prove Theorem 1.1. 


\subsection{Hyperbolic Curves of Degree 6}

Recall that a hyperbolic curve of degree 6 has three nested ovals. Bezout's Theorem implies that there are no inflection points in the smallest oval of $C$. Next theorem gives then a complete classification of possible distributions of inflection points among the ovals of a non-singular maximally inflected hyperbolic curve of degree 6 in $\mathbb{R P}^{2}$.

Theorem 1.2 Let $C$ be a non-singular maximally inflected hyperbolic curve of degree 6 in $\mathbb{R P}^{2}$. Then, the outer oval of $C$ contains at least 6 real inflection points. Moreover, for any $0 \leq k \leq 9$, there exists such a hyperbolic curve with exactly $6+2 k$ real inflection points on the outer oval.

The proof of this theorem will be given in Sect. 3 and it combines two main ingredients: First we use the tropical methods developed in [2] to construct real algebraic curves with a prescribed position of their real inflection points. Then we rely on Orevkov's braid theoretical method to prove that the outer oval of $C$ cannot contain $<6$ inflection points. Note that our proof of this latter fact uses in a crucial way that we deal with curves of degree 6 . The existence of a non-singular maximally inflected hyperbolic curve of degree $2 d>6$ with an outer oval not containing any inflection points remains an open problem.

\section{Construction of Inflected Curves}

Hilbert's method can be adapted to construct non-singular maximally inflected hyperbolic curves of even degree with all real inflection points contained in the outer oval. The proof of Theorem 1.1, given in Sect. 2.2, will consider perturbations of certain curves with generic nodes; these curves are described in Sect. 2.1. Recall that a node of an algebraic curve $C$ in $\mathbb{C P}^{2}$ is a point in the curve $C$ which is the transverse intersection of two non-singular local branches of $C$. Such a node is called generic in the case that both branches of $C$ at the point have intersection multiplicity 2 with their tangent at the node. If $C$ is a real curve with a node $p \in \mathbb{R P}^{2}$, then either $p$ is the intersection of two real branches or is the intersection of two complex conjugated branches of $C$. For our construction we shall focus only on nodes of the former type.

The fact that Hilbert's method produces maximally inflected curves is based on the following observation (see Fig. 1).

Proposition 2.1 (see [9, Proposition 3.1]) Let $C$ be a real algebraic curve in $\mathbb{R P}^{2}$ having a generic node $p \in \mathbb{R P}^{2}$ with two real branches. Then any real perturbation of $C$ has exactly two real inflection points on a neighborhood of $p$.

A real algebraic curve $C$ in $\mathbb{R P}^{2}$ is said to be generically inflected if it is nonsingular or nodal, and any of its real inflection point is a non-singular point at which $C$ has intersection multiplicity 3 with its tangent. Given a generically inflected real algebraic curve $C$, we denote by $I(C)$ the set of its non-singular real inflection points, and by $N(C)$ the set of its real generic nodes. It follows from Klein Inequality and 


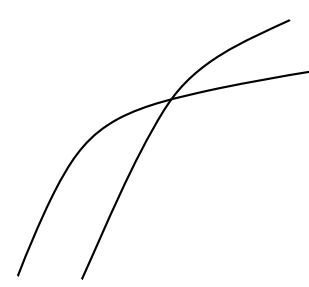

(a)

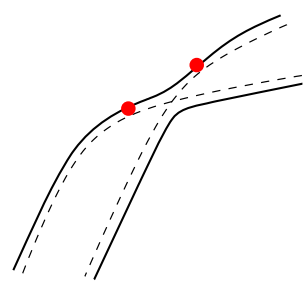

(b)

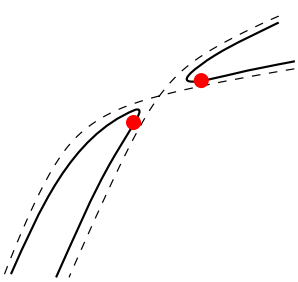

(c)

Fig. 1 Smoothing of a generic node and the creation of two real inflection points

Proposition 2.1 that if $C$ has degree $d$,

$$
\# I(C)+2 \# N(C) \leq d(d-2) .
$$

A generically inflected curve $C$ is called maximally inflected if equality holds in (*).

Next lemma is an immediate consequence of Proposition 2.1.

Lemma 2.2 Let $C$ be a generically inflected real algebraic curve in $\mathbb{R P}^{2}$. Then for any non-singular real small deformation $C^{\prime}$ of $C$, one has

$$
\# I\left(C^{\prime}\right)=\# I(C)+2 \# N(C) .
$$

In particular, any non-singular real small deformation of a maximally inflected real curve is maximally inflected.

\subsection{Construction of Hyperbolic Curves with a Maximally Inflected Outer Oval}

For the rest of this section we fix a non-singular real ellipse $C_{0}$ in $\mathbb{R}^{2}$. An oval $O$ of a maximally inflected non-singular curve $C$ is said to be maximally inflected, if $I(C) \subset O$. Let us consider the following properties for a non-singular maximally inflected hyperbolic curve $C$ of degree $2 d$ :

- $(\mathcal{P}): C$ has a maximally inflected outer oval and $\operatorname{Int}(C) \cap \operatorname{Int}\left(C_{0}\right)$ is convex;

- $\left(\mathcal{P}^{\prime}\right): C \cap C_{0}$ is a set of $4 d$ distinct real points lying on the outer oval of $C$, none of them being an inflection point of $C$.

Next we proceed by induction to construct a family $\left(C_{d}\right)_{d \geq 1}$ of real algebraic curves with the above properties of degree $2 d$ in $\mathbb{R}^{2}$. Given two real algebraic curves $C$ and $L$ in $\mathbb{R}^{2}$, respectively, defined by equations $P(x, y)=0$ and $Q(x, y)=0$, a perturbation of $C$ by $L$ is a real algebraic curve defined by the equation $P(x, y)+\varepsilon Q(x, y)=0$, with $|\varepsilon| \ll 1$ a real number. Note that two perturbations of $C$ by $L$ using parameters with opposite signs have a priori different topology in $\mathbb{R}^{2}$. The family $\left(C_{d}\right)_{d \geq 1}$ is constructed as follows:

(1) We choose for $C_{1}$ any non-singular ellipse in $\mathbb{R}^{2}$ intersecting $C_{0}$ in 4 real points. 
(2) Suppose that the curve $C_{d-1}$ is constructed, and does not contain $C_{0}$ as a component. Consider $L_{d}$ a union of $2 d$ real lines in $\mathbb{R}^{2}$ intersecting $C_{0}$ in a set $P_{d}$ of $4 d$ distinct real points such that $P_{d} \subset \operatorname{Ext}\left(C_{d-1}\right)$, and that any connected component of $C_{0} \cap \operatorname{Ext}\left(C_{d-1}\right)$ contains an even number of points of $P_{d}$. These two conditions ensure that there exists a perturbation of $C_{d-1} \cup C_{0}$ by $L_{d}$ producing a non-singular hyperbolic curve, that we define to be $C_{d}$.

Proposition 2.3 If $C_{d-1}$ satisfies properties $(\mathcal{P})$ and $\left(\mathcal{P}^{\prime}\right)$, then $C_{d}$ also satisfies properties $(\mathcal{P})$ and $\left(\mathcal{P}^{\prime}\right)$.

Proof By assumption we have

$\# I\left(C_{d-1}\right)+2 \# N\left(C_{0} \cup C_{d-1}\right)=2(d-1)(2(d-1)-2)+8(d-1)=2 d(2 d-2)$.

Hence $C_{0} \cup C_{d-1}$ is a maximally inflected real hyperbolic curve. Property $\left(\mathcal{P}^{\prime}\right)$ implies that this latter curve is generically inflected. Lemma 2.2 implies that $C_{d}$ is maximally inflected. Moreover, property $(\mathcal{P})$ implies that $I\left(C_{d-1}\right) \subset \operatorname{Ext}\left(C_{0}\right)$ and that each node of $C_{0} \cup C_{d-1}$ produces two real inflection points of $C_{d}$ on the outer oval of $C_{d}$ (see Fig. 1b). Hence $C_{d}$ has a maximally inflected outer oval, and $I\left(C_{d}\right) \subset \operatorname{Ext}\left(C_{0}\right)$.

To prove that $\operatorname{Int}\left(C_{d}\right) \cap \operatorname{Int}\left(C_{0}\right)$ is convex, it is enough to show that $\operatorname{Int}\left(C_{d}\right)$ is locally convex at each point of the outer oval of $C_{d}$ contained in $\operatorname{Int}\left(C_{0}\right)$. However this is true for points close to $P_{d}$, since $C_{d}$ is a perturbation of $C_{0}$ in a neighborhood of a point in $P_{d}$. Moreover, this is true for all points since $I\left(C_{d-1}\right) \subset \operatorname{Ext}\left(C_{0}\right)$; and hence Int $\left(C_{d}\right) \cap \operatorname{Int}\left(C_{0}\right)$ is convex.

By construction we have $C_{d} \cap C_{0}=P_{d}$ which has cardinality $4 d$. Since $I\left(C_{d-1}\right) \cap$ $C_{0}=\emptyset$, there exists a small neighbourhood $U$ of $I\left(C_{d-1}\right)$ such that $U \cap C_{0}=\emptyset$. Let $U^{\prime}$ be a neighbourhood of $P_{d-1}$ such that $P_{d} \cap U^{\prime}=\emptyset$. Then $I\left(C_{d}\right) \subset U \cup U^{\prime}$, so $C_{d}$ satisfies property $\left(\mathcal{P}^{\prime}\right)$

Note that $C_{1}$ obviously satisfies properties $(\mathcal{P})$ and $\left(\mathcal{P}^{\prime}\right)$. Hence Proposition 2.3 ensures the existence of a family $\left(C_{d}\right)_{d \geq 1}$ containing only non-singular hyperbolic curves in $\mathbb{R}^{2}$ with a maximally inflected outer oval.

\subsection{Proof of Theorem 1.1}

Here we explain how to control the numbers $\mathrm{s}\left(C_{d}\right)$ in the previous construction (see Sect. 1.1 for the definition of $\mathrm{s}(C)$ ). For this, at each step we require the additional condition that the set $P_{d}$ is disjoint from the endpoints of line segments in Hull $\left(C_{d-1} \cup C_{0}\right)$ (see item (2) in Sect. 2.1 for the definition of $\left.P_{d}\right)$. We denote by $r_{d}$ the number of points in $P_{d}$ contained in $\partial \mathrm{Hull}\left(C_{d-1} \cup C_{0}\right)$. Note that we may freely choose the number $r_{d}, d \geq 2$, between 0 and $4 d$.

Proposition 2.4 For any integer $d \geq 1$, one has $s\left(C_{d}\right)=s\left(C_{d-1} \cup C_{0}\right)$ and $s\left(C_{d} \cup C_{0}\right)=s\left(C_{d}\right)+r_{d}$.

Proof By stability, we have s $\left(C_{d}\right) \geq \mathrm{s}\left(C_{d-1} \cup C_{0}\right)$. If this inequality were strict, there would exists a line segment in $\partial$ Hull $\left(C_{d}\right)$ coming from the perturbation of a real point 
Fig. 2 Gray lines correspond to line segments in

$\partial$ Hull $\left(C_{0} \cup C_{d}\right)$. The node $n_{1}$ is a point in $P_{d}$ containted in $\partial$ Hull $\left(C_{d-1} \cup C_{0}\right)$. The node $n_{2}$ is a point of $P_{d}$ contained in the interior of Hull $\left(C_{d-1} \cup C_{0}\right)$

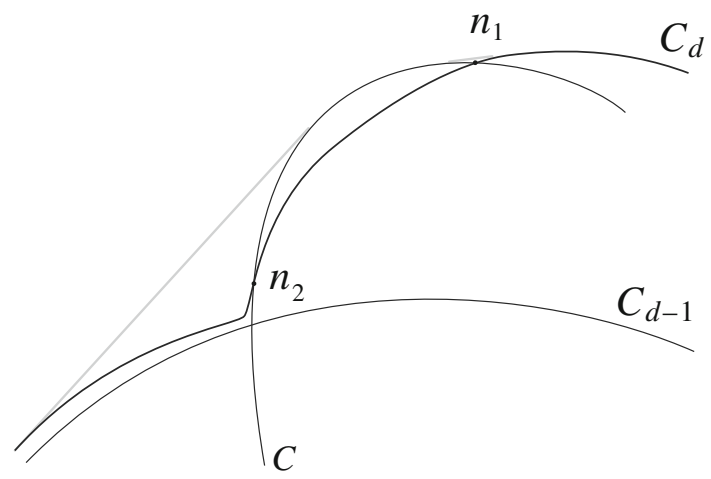

of $C_{d-1}$ at which $C_{d-1}$ has intersection multiplicity at least 4 with its tangent. However the curve $C_{d-1}$ is maximally inflected, and in particular is generically inflected. Hence all this together proves that $\mathrm{s}\left(C_{d}\right)=\mathrm{s}\left(C_{d-1} \cup C_{0}\right)$.

There are two types of line segments contained in $\partial \mathrm{Hull}\left(C_{d} \cup C_{0}\right)$ : those which are a perturbation of a line segment contained in $\partial \mathrm{Hull}\left(C_{d-1} \cup C_{0}\right)$, and those which come from the perturbation of $C_{d-1} \cup C_{0}$ in a neighborhood $U_{p}$ of a point $p \in$ $P_{d} \cap \partial \operatorname{Hull}\left(C_{d-1} \cup C_{0}\right)$; see Fig. 2. The set $\partial$ Hull $\left(\left(C_{d} \cup C_{0}\right) \cap U_{p}\right)$ contains a line segment. Hence the set $\partial$ Hull $\left(C_{d} \cup C_{0}\right)$ contains exactly $r_{d}$ line segments more than วHull $\left(C_{d-1} \cup C_{0}\right)$.

Now, Theorem 1.1 follows from the fact that we can choose the number $r_{d}$ between 0 and $4 d$ freely, and because $\mathrm{s}\left(C_{1}\right)=0$ and $\mathrm{s}\left(C_{1} \cup C_{0}\right)=4$.

Example 2.5 Let us give an explicit equation of a degree 6 polynomial defining a nonsingular hyperbolic curve of degree 6 in $\mathbb{R}^{2}$ with 12 line segments on the boundary of its convex hull. For that, consider three numbers $\varepsilon_{1}=1.58, \varepsilon_{2}=-5.5 \times 10^{-3}$ and $\varepsilon_{3}=-10^{-10}$, and denote by $C:=C(x, y)=x^{2}+y^{2}-1$. Consider the following polynomial:

$$
f(x, y):=C^{3}+C^{2}\left(y^{2}-a^{2} x^{2}\right) \varepsilon_{1}+C\left(y^{2}-x^{2}\right)\left(y^{2}-b^{2} x^{2}\right) \varepsilon_{2}+\varepsilon_{3},
$$

where $a=\tan (\pi / 12)$ and $b=\tan (5 \pi / 12)$. Figure 3 is a numerical plot of the zero locus of $f$. In the construction of this example we used two lines through the origin at angles $\pm \frac{\pi}{12}$ in the first step, and four lines through the origin at angles $\pm \frac{\pi}{4}, \pm \frac{5 \pi}{12}$ in the second step (all angles are measured with respect to the positive $x$-axis). The plot in Fig. 3 is obtained by finding numerically the roots of the real one variable polynomial defined by the intersection of the curve with lines through the origin.

\section{Maximally Inflected Hyperbolic Curves of Degree 6}

Now we turn to the study all possible arrangements of the 24 real inflection points of a non-singular maximally inflected hyperbolic sextic $C$ in $\mathbb{R P}^{2}$. We denote by 
Fig. 3 A non-singular hyperbolic curve of degree 6 in $\mathbb{R}^{2}$ with exactly 12 line segments in the boundary of its convex hull. Shaded regions on the outer oval (three in each quadrant) correspond to points in the curve contained in the interior of the convex hull of the curve

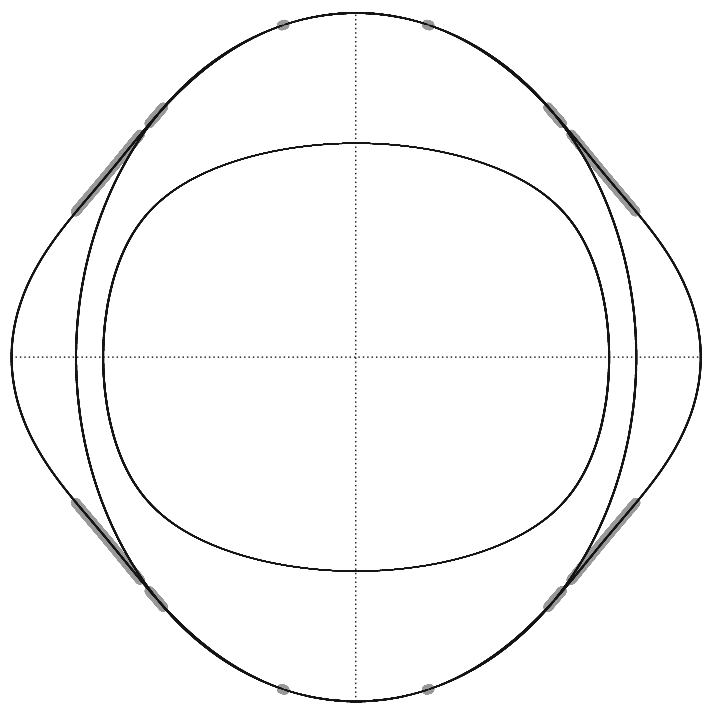

$O_{1}, O_{2}, O_{3}$ the three ovals of $C$ in such a way that $O_{1}$ contains $O_{2}$ which in its turn contains $\mathrm{O}_{3}$. By Bezout Theorem, the oval $\mathrm{O}_{3}$ does not contain any real inflection point. Moreover, each oval $O_{1}$ and $O_{2}$ contains an even number of real inflection points.

\subsection{Obstructions}

Here we prove that the oval $\mathrm{O}_{2}$ cannot contain more than 18 real inflection points. Proposition 3.1 is a straightforward consequence of Lemmas 3.3 and 3.2, that we prove in the rest of this section.

Proposition 3.1 Let $C$ be a non-singular maximally inflected algebraic hyperbolic curve of degree 6 in $\mathbb{R P}^{2}$. Then the oval $O_{1}$ contains at least 6 real inflection points.

Let $C_{0}$ and $C_{1}$ be two non-singular real algebraic curves in $\mathbb{R P}^{2}$ of degree at least 3 , such that there exists a continuous path $\left(C_{t}\right)_{0 \leq t \leq 1}$ of non-singular real algebraic curves in $\mathbb{R P}^{2}$ from $C_{0}$ to $C_{1}$. A real line $D$ is said to be deeply tangent to the curve $C_{t}$ if there exists a point $p \in D \cap C_{t}$ such that the order of contact of $C_{t}$ and $D$ is at least 4.

Recall that if $C_{0}$ and $C_{1}$ are generic, then they do not have any deep tangent lines. Moreover Klein proved in [5] (see also [9]) that in this case, it is always possible to choose the family $\left(C_{t}\right)$ such that appearance and disappearance of real inflections points when $t$ varies occur only when passing through a curve $C_{t_{0}}$ with a unique deep tangent $D$ with order of contact exactly 4 with $C_{t_{0}}$ at a unique point $p$ (see Fig. $4 \mathrm{~b}$ ). Then for $t=t_{0}+\varepsilon$ with $-1 \ll \varepsilon \ll 1$ a non-zero real number, the line $D$ deforms to a real bitangent of $C_{t}$ tangent to $C_{t}$ in two real points close to $p$ (see Fig. $4 \mathrm{a}$ ), and deforms to a real bitangent of $C_{t}$ tangent to $C_{t}$ to two complex conjugated points for 


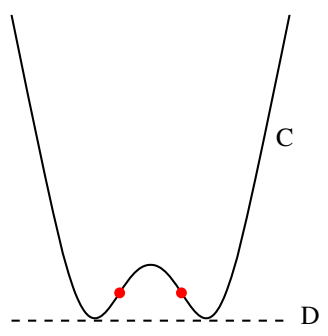

(a) $C=C_{t_{0}+\varepsilon}$

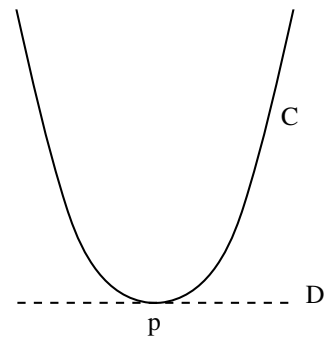

(b) $C=C_{t_{0}}$

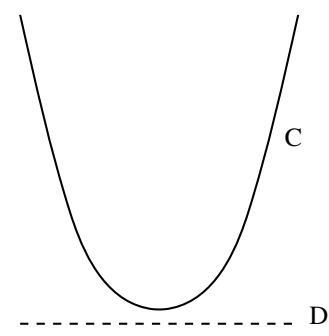

(c) $C=C_{t_{0}-\varepsilon}$

Fig. 4 Appearance/disappearance of real inflection points

$t=t_{0}-\varepsilon$ (see Fig. 4c). The curve $C_{t_{0}+\varepsilon}$ has two real inflection points close to $p$, that disappear when passing through $C_{t_{0}}$.

Lemma 3.2 Let $C$ be a non-singular maximally inflected hyperbolic curve of degree 6 in $\mathbb{R P}^{2}$ such that the oval $O_{i}$ contains exactly $2 n$ real inflection points. Then the curve $C$ has exactly $n$ real bitangents which are tangent to $C$ at two points on $O_{i}$.

Proof Let $C_{0}$ be the non-singular hyperbolic real sextic in $\mathbb{C P}^{2}$ obtained by a generic perturbation of the union of 3 nested disjoint ellipses. By construction, $C_{0}$ does not have any real inflection points, and does not have any real bitangent tangent to two real points.

It follows from Bezout Theorem that the number of real bitangents is invariant under deformation in the space of non-singular real algebraic curves of degree 6 . Since the space of non-singular hyperbolic sextics in $\mathbb{R P}^{2}$ is connected (see [6]), the result follows from the description of appearance/disappearance of real inflection points given above.

Lemma 3.3 Let $C$ be a non-singular maximally inflected hyperbolic curve of degree 6 in $\mathbb{R P}^{2}$ with exactly $n$ real bitangents tangent to $C$ at two points on $\mathrm{O}_{2}$ (see Fig. $5 a$ ). Then $n \leq 9$.

Proof The proof uses the symplectic techniques developed by Orevkov to study the topology of real plane curves. For the sake of shortness, we do not recall this technique here. We refer instead to [8, Sect. 2.1] for the definition of a real pseudoholomorphic curve in $\mathbb{C P}^{2}$, and to [7, Sect. 3] for the definition of a $\mathcal{L}_{p}$ scheme and for an account of the braid theoretical methods in the study of real plane curves. Note nevertheless that any real algebraic curve is a real pseudoholomorphic curve.

Choose a point $p$ inside the oval $O_{3}$. Applying [7, Proposition 3.6] if necessary, this implies that the $\mathcal{L}_{p}$-scheme depicted in Fig. $5 \mathrm{~b}$ is realizable by a reducible real pseudoholomorphic curve of degree $6+n$, whose irreducible components are a sextic $C^{\prime}$ and $l$ real pseudoholomorphic lines $D_{1}, \ldots, D_{n}$ intersecting $C^{\prime}$ transversely in two points and tangent to $C^{\prime}$ in two points. We emphasize that we do not claim that the union of $C$ with its real bitangent to $O_{2}$ realizes the $\mathcal{L}_{p}$-scheme depicted in Fig. 5b. This is precisely the point where we need to consider real pseudholomorphic curves instead of real algebraic curves, since [7, Proposition 3.6] does not hold for those latter. 


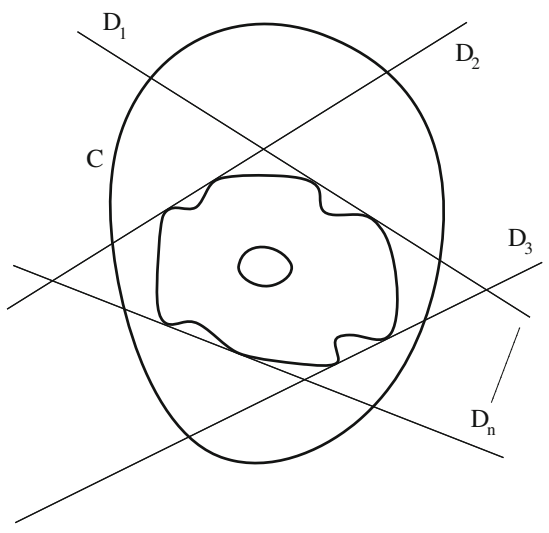

(a)

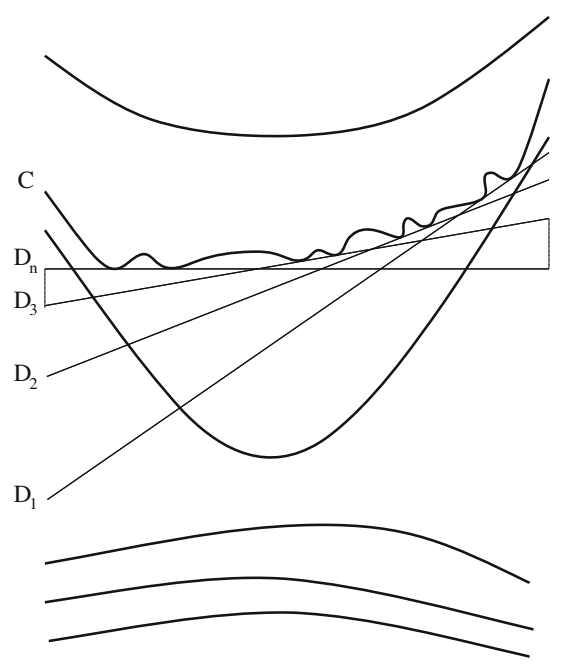

(b)

Fig. 5 A hyperbolic sextic together with bitangents

The braid associated to this $\mathcal{L}_{p}$-scheme is

$$
b_{n}=\prod_{i=4}^{n+3}\left[\left(\prod_{j=i}^{n+3} \sigma_{j}^{-1}\right) \sigma_{n+4}^{-4}\right] \cdot \prod_{j=4}^{n+3} \sigma_{j}^{-1} \cdot \prod_{i=1}^{n+5}\left(\prod_{j=i}^{n+5} \sigma_{j}\right) .
$$

Computing the Alexander polynomial $p_{10}$ of $b_{10}$, we see that $\pm i$ is a simple root of $p_{10}$. On the other hand the sum of exponents of $b_{10}$ equals 15 , which is the number of strings of $b_{10}$ minus 1. Hence the Murasugi-Tristram inequality [7, Sect. 2.4] together with [7, Lemma 2.1] implies that the $\mathcal{L}_{p}$-scheme depicted in Fig. 5b with $n \geq 10$ is not realizable by a real pseudoholomorphic curve of degree $6+n$.

Proof of Proposition 3.1 Let $2 n$ be the number of real inflection points of $C$ contained in $\mathrm{O}_{2}$. According to Lemma 3.2 the curve $C$ has $n$ real bitangents which are tangent to $C$ at two points on $O_{2}$. Hence we get $n \leq 9$ by Lemma 3.3.

\subsection{Constructions}

We end the proof of Theorem 1.2 by showing that all distributions of the 24 inflection points on the ovals $O_{1}$ and $O_{2}$ which are not forbidden by Proposition 3.1 are realizable.

Proposition 3.4 For any integer $0 \leq k \leq 9$, there exists a non-singular maximally inflected hyperbolic curve $C(k)$ of degree 6 in $\mathbb{R P}^{2}$ such that $O_{1}$ contains exactly $6+2 k$ real inflection points.

Proof The curve $C(9)$ has been constructed in Sect. 2.1. The curve $C(k)$ with $k=$ $1,3,5$ and 7 is constructed similarly. Consider two real conics $C_{0}$ and $C_{1}$ intersecting 


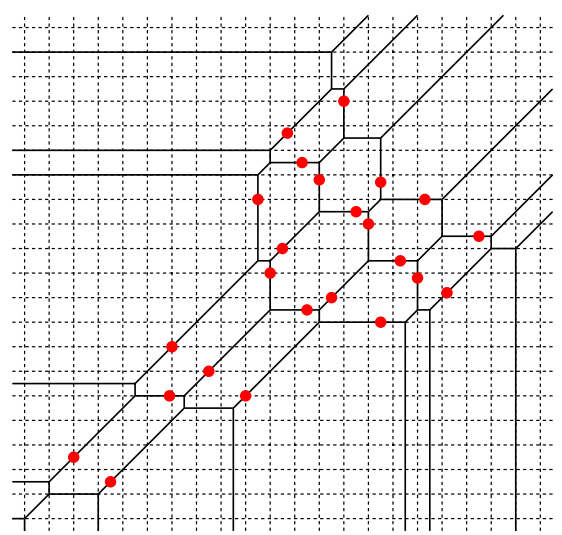

(a) $\mathbb{T} C$

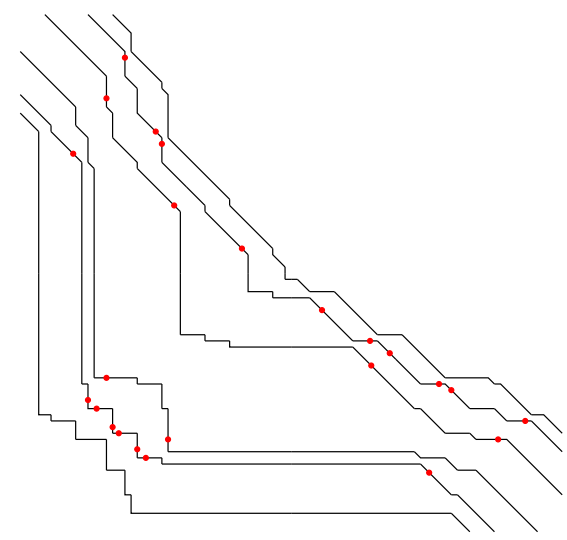

(b)

Fig. 6 Patchworking of the curve $C(0)$

into 4 real points. Choose $\frac{k-1}{2}$ lines intersecting $C_{0}$ in $k-1$ points in $\operatorname{Ext}\left(C_{0} \cup C_{1}\right)$, and $\frac{7-k}{2}$ lines intersecting $C_{0}$ in $7-k$ points in Int $\left(C_{0} \cup C_{1}\right)$. Denote by $L$ the union of these lines. We obtain a non-singular maximally inflected quartic $C_{2}$ by perturbing $C_{0} \cup C_{1}$ with $L$. By perturbing $C_{0} \cup C_{2}$ to a non-singular maximally inflected curve of degree 6 , we obtain the curve $C(k)$.

We construct the curves $C(k)$ with $k$ even by patchworking. We refer to [2] for the definition of tropical inflection points of tropical plane curves, and for the construction of real algebraic curves with a controlled position of their real inflection points by patchworking. The tropical curve $\mathbb{T} C$ depicted in Fig. 6a together with the patchworking depicted in Fig. 6b produce the curve $C(0)$. The red dots represent inflection points in Fig. 6 (note that all tropical inflection points have complex multiplicity 3, and real multiplicity 1 in this case). Since their position on the tropical curve heavily depends on the length of its edges, we depicted $\mathbb{Z}^{2} \subset \mathbb{R}^{2}$ by the intersection points of the doted lines in Fig. 6a.

Let us recall briefly how to locate real inflection points in Fig. 6b. To this aim we use the ribbon interpretation of Patchworking, for which one can refer for example to [1, Sect. 3]. The Patchworking depicted in Fig. 6b is obtained by twisting all bounded edges of $\mathbb{T} C$. We depict in Fig. 7a the intermediate picture between Fig. 6a and b. The ovals $O_{1}, O_{2}$, and $O_{3}$ of $C(0)$ correspond respectively to the blue, purple, and green curves. Note that Fig. 7a represents the logarithmic image of $C(0)$, in particular the position of $C(0)$ with respect to its tangent lines cannot be read directly on Fig. 7a. A local computation gives this position for an arc whose logarithmic image is close to a vertex of $\mathbb{T} C$ which is not locally a tropical line. One gets in this way the location of real inflection points corresponding to tropical inflection points located at vertices of $\mathbb{T} C$ (there are no such tropical inflection points in this particular example, however we are describing the general procedure to recover the location of real inflection points in a patchworking). This partial knowledge of the position of $C(0)$ with respect to its tangent lines determines in its turn the location of real inflections points corresponding to tropical inflection points located on edges of $\mathbb{T} C$. For example, any arc whose 


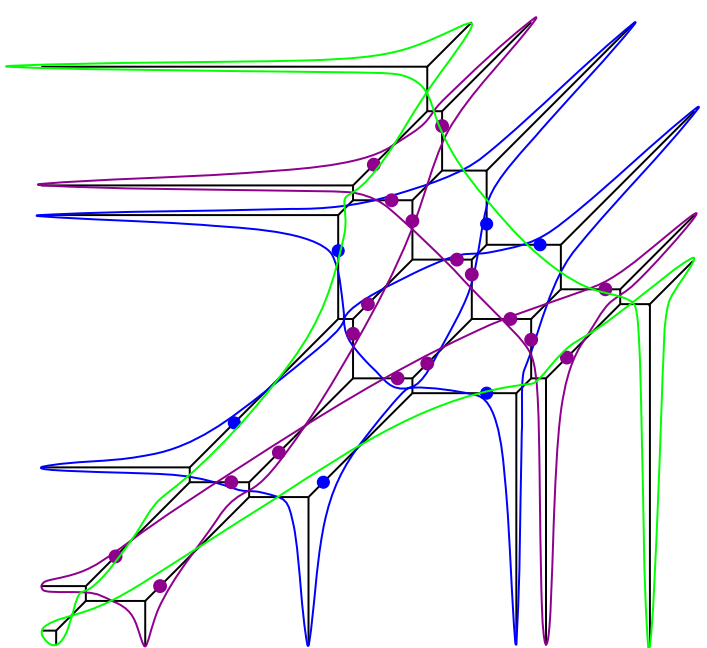

(a)

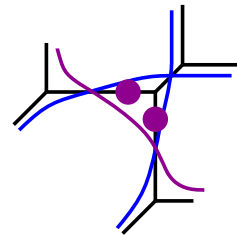

(b)

Fig. 7 Patchworking of the curve $C(0)$, intermediate step (each tropical inflection point is coloured according to the arc it comes from)

logarithmic image is depicted in Fig. 7b must contain an even number of real inflection points. Hence these two latter must be located on the only arc whose logarithmic image passes close to the two tropical inflection points.

By varying the length of the edges of $\mathbb{T} C$, we construct similarly the curves $C(2)$, $C(4)$, and $C(6)$. Note that any bounded edge of $\mathbb{T} C$ adjacent to an unbounded component of $\mathbb{R}^{2} \backslash \mathbb{T} C$ corresponds to a green arc, which is the logarithmic image of a (convex) arc of $\mathrm{O}_{3}$. In particular for any choice of the length of edges of $\mathbb{T} C$, this construction produces a real hyperbolic curve with at least six real inflection points on both $\mathrm{O}_{1}$ and $\mathrm{O}_{2}$.

The construction of the curve $C(8)$ requires a mild adaptation of the method presented in [2]. Consider the tropical curve $\mathbb{T} C(8)$ depicted in Fig. 8a. Note that $\mathbb{T} C(8)$ has a node: all its vertices are 3 -valent, except one 4-valent vertex $p$ which is the transversal intersection of two edges of $\mathbb{T} C(8)$. The method used in [2] to locate tropical inflexion points and to compute their real and complex multiplicities only uses local data about tropical curves. Since the two edges of $\mathbb{T} C(8)$ intersecting at $p$ do not have any common direction with an edge of a tropical line, this implies that all computations performed in [2] to locate and compute the multiplicities of tropical inflexion points of $\mathbb{T} C(8)$ distinct from $p$ still holds in this case. In particular, red dots in Fig. 8 a represent tropical inflexion points distinct from $p$ with real multiplicity 1 , and blue dots those of real multiplicity 0. Using Shustin's version [11] of Viro's Patchworking Theorem to construct singular curves, we can approximate the real tropical curve depicted in Fig. 8 b by a family $\left(\widetilde{C}_{t}\right)_{t \gg 1}$ of real hyperbolic sextic with one generic node. Up to a translation in $\mathbb{R}^{2}$, the local equation of $\widetilde{C}_{t}$ at the node is 


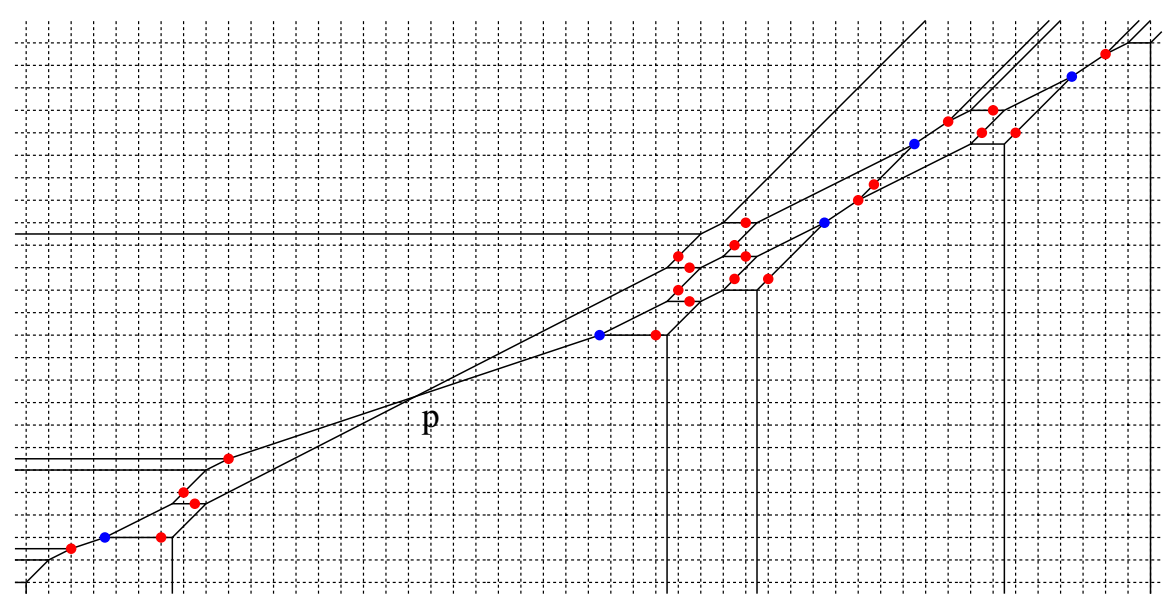

(a)

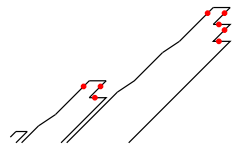

Fig. 8 Patchworking of the curve $\widetilde{C}(8)$

$$
\left(x-\alpha y^{2}\right)\left(x+\beta y^{3}\right)+o_{t \rightarrow+\infty}\left(t^{-1}\right)
$$

with $\alpha$ and $\beta$ two positive real numbers. Proposition 2.1 implies that smoothing this node such that the resulting curve is hyperbolic produces the curve $C(8)$.

Remark 3.5 If one smoothes "tropically" the node of the tropical curve $\mathbb{T} C$ (8) (i.e. perturbing $p$ into two 3 -valent vertices before patchworking), the two additional real inflection points appear on the oval $\mathrm{O}_{2}$ in the positive quadrant of $\mathbb{R}^{2}$ instead of appearing on the oval $O_{1}$ in the quadrant $\{x>0, y<0\}$. This is a manifestation of the totally discontinuous topology induced on a field by a non-archimedean valuation.

On the other hand, one can use original Viro's Patchworking to construct directly the non-singular curve $C(8)$. In this case, one should use the chart (in the sense of [12]) at the node $p$ of $\mathbb{T} C(8)$ given by a suitable perturbation of the curve $\left(x-y^{2}\right)\left(x+y^{3}\right)$. 
Acknowledgments We thank the Laboratorio Internacional Solomon Lefschetz (LAISLA), associated to the CNRS (France) and CONACYT (Mexico). The first author was partially supported by CONACyTMéxico \#167594. The third author was partially supported by UNAM-PAPIIT IN-117110. We also thank Bernd Sturmfels for his stimulating questions, and, as usual, Jean-Jacques Risler for valuable comments on a preliminary version of this paper.

\section{References}

1. Brugallé, E.: Tropical curves, notes from introductary lectures given in July 2013 at Max Planck Institute for Mathematics, Bonn. http://erwan.brugalle.perso.math.cnrs.fr/articles/TropicalBonn/ TropicalCurves

2. Brugallé, E., López de Medrano, L.: Inflection points of real and tropical plane curves. J. Singul. 4, 74-103 (2012)

3. De Loera, J., Sturmfels, B., Vinzant, C.: The central curve in linear programming. Found. Comput. Math. 12(4), 509-540 (2012)

4. Kharlamov, V., Sottile, F.: Maximally inflected real rational curves. Mosc. Math. J. 3(3), 947-987, 1199-1200 (2003)

5. Klein, F.: Über den Verlauf der Abelschen Integrale bei den Kurven vierten Grades. Math. Ann. 10, 365-397 (1876)

6. Nuij, W.: A note on hyperbolic polynomials. Math. Scand. 23, 69-72 (1968)

7. Orevkov, S.Y.: Link theory and oval arrangements of real algebraic curves. Topology 38(4), 779-810 (1999)

8. Orevkov, S.Y.: Classification of flexible $M$-curves of degree 8 up to isotopy. Geom. Funct. Anal. 12(4), 723-755 (2002)

9. Ronga, F.: Klein's paper on real flexes vindicated. In: Pawlucki, W., Jakubczyk, B., Stasica, J. (eds.) Singularities Symposium-Lojasiewicz 70, vol. 44. Banach Center Publications, Warsaw (1998)

10. Schuh, F.: An equation of reality for real and imaginary plane curves with higher singularities. Proc. Sect. Sci. R. Acad. Amst. 6, 764-773, 1903-1904

11. Shustin, E.: Gluing of singular and critical points. Topology 37(1), 195-217 (1998)

12. Viro, O.Y.: Gluing of plane real algebraic curves and constructions of curves of degrees 6 and 7. In: Topology (Leningrad, 1982), Lecture Notes in Mathematics, vol. 1060, pp. 187-200. Springer, Berlin (1984)

13. Viro, O.Y.: Some integral calculus based on Euler characteristic. In: Lecture Notes in Mathematics, vol. 1346, pp. 127-138. Springer, Berlin (1988)

14. Viro, O.Y.: Real plane algebraic curves: constructions with controlled topology. Leningr. Math. J. 1(5), 1059-1134 (1989) 